\title{
Concordances linguistiques entre aires celtique et romane à partir des données des atlas multilingues
}

Linguistic concordances between Celtic and Romance areas based on multilingual atlas data

Daniel Le Bris et Elisabetta Carpitelli

\section{OpenEdition}

\section{Journals}

Édition électronique

URL : https://journals.openedition.org/lbl/1137

DOI : $10.4000 / \mathrm{lbl} .1137$

ISSN : 2727-9383

\section{Éditeur}

Université de Bretagne Occidentale - UBO

\section{Édition imprimée}

Date de publication : 1 mars 2014

Pagination : 73-85

ISBN : 979-10-92331-07-3

ISSN : $1270-2412$

\section{Référence électronique}

Daniel Le Bris et Elisabetta Carpitelli, « Concordances linguistiques entre aires celtique et romane à partir des données des atlas multilingues », La Bretagne Linguistique [En ligne], 18 | 2014, mis en ligne le 01 mai 2021, consulté le 21 mai 2021. URL : http://journals.openedition.org/lbl/1137 ; DOI : https:// doi.org/10.4000/lbl.1137

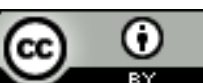

La Bretagne Linguistique est mise à disposition selon les termes de la Licence Creative Commons Attribution 4.0 International. 


\section{Concordances linguistiques entre aires celtique et romane à partir des données des atlas multilingues}

\section{L'Atlas Linguarum Europae et l'Atlas Linguistique Roman dans le cadre de la théorie de la motivation lexicale}

\footnotetext{
$\mathrm{C}_{\mathrm{s}}$

es deux grandes entreprises atlantographiques multilingues la première à l'échelle continentale et la deuxième à l'échelle d'un domaine linguistique homogène - sont nées, d'une part, d'une exigence ressentie par les dialectologues européens, et de manière plus générale par les linguistes, de commencer à exploiter la grande masse de données cartographiques accumulées depuis la fin du XIX ${ }^{\mathrm{e}}$ siècle ; d'autre part, de la mise en place d'un réseau stable de coopération internationale avec des chercheurs restés parfois longtemps isolés à cause des aléas de la politique européenne de leurs pays respectifs ${ }^{1}$.

On considère souvent l'ALE et l'ALiR comme deux entités liées par un rapport de filiation pour des raisons historiques, structurelles mais également théoriques :

a) l'Atlas Linguistique Roman (ALiR) naît d'abord de l'initiative

* Maître de conférences de celtique, CRBC (EA 4451/ UMS 3554), UBO/ueb.

** Professeur de sciences du langage, BCL (UMR 7320), UNS.

1. N'oublions pas que la fondation de l'Atlas Linguarum Europae se situe à l'époque où l'Europe était encore divisée en deux blocs nettement opposés.
} 
de dialectologues qui ont collaboré depuis l'origine à l'Atlas Linguarum Europae (ALE) ;

b) le réseau de localités est fondé dans les deux atlas à partir de points et de «cases». Ces dernières contiennent plusieurs points lorsque différentes sources atlantographiques existent : notamment nationales et régionales (cf. Italie, France, Galice) ;

c) l'ALE et l'ALiR sont des atlas interprétatifs car ils synthétisent et représentent le résultat d'une analyse. Ils ne sont donc pas de simples atlas de données brutes, même si dans certains cas, des enquêtes $a d$ hoc ont dû être faites pour des domaines encore dépourvus d'un atlas linguistique (Portugal et Grèce) ;

d) les deux publications prévoient une cartographie «à symboles» accompagnée de commentaires ;

e) la cartographie motivationnelle est privilégiée par rapport à la cartographie onomasiologique : le linguiste montre donc la diffusion aréale des représentations sur lesquelles se fonde la création lexicale plutôt que la diffusion de types lexicaux différents.

Mario Alinei, cofondateur de l'ALE, a développé l'approche de la motivation sémantique à partir de la carte et du commentaire des désignations européennes de l'arc-en-ciel (Alinei, 1983). Cette approche formule l'hypothèse que tous les signes linguistiques naissent motivés. Mais, les motivations originelles peuvent être effacées et substituées par des motivations nouvelles, lorsque des changements culturels importants se produisent dans la communauté des locuteurs. Selon cette hypothèse, dans le domaine de la zoonymie, de la phytonymie, des noms de phénomènes atmosphériques et des maladies, l'évolution lexicale se corrèle étroitement à l'évolution religieuse. Cette dernière a un impact sur les représentations de l'univers révélées par le lexique (Alinei, 2009). Les concordances motivationnelles entre types lexicaux très différents, relatifs à ces champs sémantiques et répertoriés dans des aires linguistiques éloignées et génétiquement non apparentées - comme c'est le cas dans 1'ALE - pourraient témoigner d'un stade préhistorique commun à l'ensemble du continent ${ }^{2}$.

2. L'intérêt pour les concordances entre aires linguistiques génétiquement hétérogènes se corrélait depuis la fondation du chantier de l'ALE avec une attention plus générale à la question des universaux linguistiques : «L'Atlas Linguistique 
Cette approche a été ainsi résumée par Alinei (1994 : 20-21) dans un passage qui révèle aussi un engagement humaniste de la part des linguistes participant à l'atlas européen :

«Ce qui nous a poussés à la réalisation de l'ALE [...] a été $[\ldots]$ le sentiment $[\ldots]$ de l'universalité de l'homme, le besoin de découvrir une identité plus profonde $[\ldots]$ qui n'ignore pas les différences, mais qui, au contraire, les reconnaisse, mais reconnaisse aussi $[\ldots]$ les ressemblances profondes ${ }^{3} . »$

Les interprétations des désignations européennes, en particulier celles de l'arc-en-ciel (Alinei, 1983), de la belette (Alinei, 1986) et de la coccinelle (Alinei et Barros Ferreira, 1994), ont inauguré une réflexion sur la stratigraphie culturelle des motivations magicoreligieuses émergeant du lexique dialectal européen qui avait l'ambition, d'après Alinei (1994 : 27), de permettre une «élévation des datations linguistiques». La catégorie motivationnelle considérée comme la plus ancienne, révélée par des zoonymes qui désignent par exemple des phénomènes atmosphériques, des maladies ou des parties du corps humain, correspondrait à un cadre de croyances ancestrales, communes à toutes les communautés du continent. Alinei fait remonter ces croyances au Paléolithique moyen et supérieur. L'image de l'arc-en-ciel, identifié à un buveur, apparaît dans des formes telles que l'italo-roman septentrional arco bevente «arc buvant», avec une représentation que l'on retrouve aussi dans le roumain curcubeu, dans l'oudmourte [vuju'is'] (cf. également le lat. ARCUS BIBIT), mais aussi dans l'ukrainien sivarvanja "pompe», le lituanien straublys «trompe (qui suce)»(Alinei, 1983, 68-69).

de l'Europe trouve sa raison d'être en fournissant des données linguistiques sur un très vaste territoire : le continent européen. Ces matériaux [...] doivent permettre, par une nouvelle méthode de comparaison, plus «horizontale» que celle permise par les autres atlas, de franchir, par principe, les frontières entre les langues et les familles de langues, et d'envisager ainsi les vieux problèmes tels que la parenté entre les langues, les phénomènes de l'analogie de langues non-apparentées, l'interférence, les universaux, la typologie linguistique...» (KRUIJSEN, 1977 [1974] : 383).

3. «[...] ciò che ha mosso noi alla realizzazione dell'ALE $[\ldots]$ è stato [...] il sentimento $[\ldots]$ dell'universalità dell'uomo, il bisogno di scoprire un'identità più profonda $[\ldots]$ che non ignori le differenze, ma anzi le riconosca, ma riconosca anche $[\ldots]$ le profonde somiglianze». 
Elle correspond à une représentation du phénomène semblable à un animal qui aspire l'eau de la mer et de la terre pour la restituer sous forme de pluie. Cette mythologie préhistorique à caractère totémique est également présente dans les images du «serpent arc-en-ciel», connues dans certaines cultures ethnographiques très éloignées de l'Europe (Benozzo, 2007 ; Lanaia, 2007), où les images des éléments de l'univers correspondent souvent à des représentations animalières. D'autres désignations, comme l'ibéro-roman arco (ou risco ou encore circo) da vella renvoient à des anthropomorphismes. Elles révèlent une fois de plus cette vision totémique de la nature : une forme particulière de respect pour un phénomène ou un être impressionnant induit la communauté à les tabouiser et à tabouiser par là-même leurs noms. On esquive ainsi le mauvais sort en remplaçant le nom de la chose crainte par une autre dénomination. Il se peut que la communauté des locuteurs aille jusqu'à considérer le phénomène ou l'être désigné comme un animal protecteur de la famille, comme cela se vérifie dans le slovène mavrica ou le catalan ase de sant marti (Alinei, $1983: 68$ ).

Ainsi, la reconstruction linguistique se corrèle de manière très étroite à une reconstruction des strates culturelles et religieuses qui se sont succédé dans le continent, selon une évolution où les désignations animalières et parentélaires des éléments de l'univers seraient les plus anciennes. Ces désignations sont d'abord associées à des dénominations païennes qui renvoient soit à des divinités comme Iris, Venus ou Ukko, soit à des animaux-dieux (ou encore à des dons destinés à ces personnages) ${ }^{4}$. Dans un second temps, leurs dénominations peuvent renvoyer à une religion plus moderne, monothéiste. C'est le cas, par exemple, des nombreuses formes galloromanes qui contiennent le nom d'un saint, de la vierge ou de Dieu (pont de Saint Bernard, ruban de la Vierge, alliance du bon dieu, etc.). Les désignations qui apparaissent comme les plus descriptives

4. «Centaurs, mermaids, harpies, Gorgons, and hundreds of other half-animal, halfhuman monsters and gods, are typical, frozen forms of [the] transition. [...] Also names such as Mac[edonian] vinožito "wine and corn", [v'ini i rakija] "wine and liquor" and the many similar names [...] like It[alian] "wine and bread", Alb[anian] "oil, wine and vinegaré", etc., belong to this group, since they reflect the belief that, depending on their intensity, the colours of the rainbow [...] announce good crops» (AlineI, $1983: 52$ ). 
- italo-roman sette colori, gallo-roman godet de couleurs ou cent couleurs - doivent être considérées comme appartenant à un cadre culturel désormais laïcisé.

Dans cette perspective stratigraphique, les recherches conduites sur la motivation lexico-sémantique dans un domaine linguistique homogène étaient a priori perçues comme moins intéressantes, par rapport au résultat escompté, dans un espace aussi génétiquement diversifié que celui de l'Europe : «Pour des raisons théoriques qui seraient trop longues à expliquer, l'étude des motivations n'est pas très productive au sein du même groupe linguistique» (Alinei, 1994, $25)^{5}$.

Contrairement à cette prévision, l'ALiR, chantier international présenté lors d'une réunion du Département Roman de l'ALE, à Aussois en 1986, par Gaston Tuaillon et Michel Contini (Université de Grenoble), s'est révélé extrêmement productif, notamment en fonction de la reconstruction étymologique. Dalbera (Gadet, 2009, 132) souligne le principe selon lequel la variation dans l'espace est une projection de la variation dans le temps. Il précise aussi l'objectif de l'ALE et de l'ALiR :

«Les dialectologues collaborent actuellement à des atlas [...] interprétatifs, ALE et ALiR, dans un programme de lecture et commentaire des cartes de synthèse élaborées à partir des atlas dialectaux. L'objectif est de dégager des constantes dans la motivation des types lexicaux, qui constitueraient des signifiés primitifs. [...] À l'échelle de l'Europe, cela conduit à une vision typologique des motifs ; à l'échelle de la Romania, cela se double d'une perspective génétique et rejoint l'étymologie [...] : étymologies élucidées, récurrences motivationnelles massives.»

Dalbera émet l'hypothèse suivante : un motif apparaissant comme constant dans l'espace, au-delà des frontières entre plusieurs domaines linguistiques, et cela malgré les différences lexicales, pourrait correspondre à la représentation la plus stable dans le temps, et serait par là-même la plus ancienne. Il ne se contente donc pas de l'étymologie phonétique : il essaie toujours de reconstruire

5. «Per ragioni teoriche che sarebbe troppo lungo spiegare, lo studio delle motivazioni non produce molto nell'ambito dello stesso gruppo linguistico. » 
le sens d'un étymon parallèlement au signifiant et par la méthode comparative $^{6}$.

\section{Études de zoonymes celtiques et romans}

Notre recherche utilise les données de l'ALF, des atlas régionaux de France, de l'AIS, de l'ALI, de l'ALE et de l'ALiR ${ }^{7}$. Dans le cadre de cet article, plusieurs analyses sont établies à partir de sources maritimes: l'Atlas linguistique de la faune maritime de Bretagne (ALFMB) (Le Berre, 1986 \& 2008), les Espèces marines de Normandie (Lepelley, 2005), la Nomenclatura de la flora y fauna maritimas de Galicia (Ríos Panisse, 1977-1983), le Léxico de los marineros peninsulares (LMP) (Alvar, 1985-1989) et l'Atlas linguístico do litoral português (ALLP) (Vitorino, 1985-...).

L'analyse motivationnelle des désignations romanes du ver de terre a dévoilé une représentation dominante, consistant en une partie allongée interne ou externe du corps humain comme les boyaux, ou plus précisément les intestins ou encore le cordon ombilical : cf. casentaru (et variantes) de l'Italie du sud qui signifie littéralement «entrailles de la terre», viskoli terme italo-roman méridional, signifiant «viscères». Ajoutons encore les nombreux aboutissants du lat. LUMBRICUS, si l'on accepte l'hypothèse récente d'Alinei (2010a et 2010b) qui considère ce mot comme une évolution d'UMBILICUS «nombril» avec l'article agglutiné (Alinei, 2010b) ${ }^{8}$. L'ensemble de ces images de parties allongées du corps apparaît comme une série

6. «La reconstruction comparative des signifiés [...] renvoie à une perspective cognitive par l'éclairage sur les modes de création et le renouvellement du lexique [...]» (DALBERA, in Gadet 2009 : 134).

7. ALF : Atlas Linguistique de France ; AIS : Atlante Italiano-Svizzero ; ALI : Atlante Linguistico Italiano.

8. «[...] the AIS map 457 ("il lombrico") shows a development which is crucial for our thesis: in a vast northern-central area, derivates of lumbricus appear with what in the traditional framework would appear as the 'deglutination'of the article - that is to say, the wrong interpretation of initial /1/ as an article, and its consequent elimination -: Italian lombrico, 'earthworm', is transformed in l'ombrico, 'the earthworm'. [...] the same data can be interpreted in exactly the reverse way: it is the original Latin form *umbricus 'navel', born as a dialectal development of umbilicus, which has been preserved, and has become, with the 'agglutination' of the article, the name of the earthworm lumbricus.» (ALINEI, 2010b : 6). 
cohérente qui pourrait permettre d'élucider une désignation occitane de cet animal, [boð'ik] (et variantes), dont l'étymon demeure inconnu (FEW 21, 279b). Une série de formes rattachées au lat. BOTELLUS «boyau», "intestins», telles que bot «outre», boudigue "cornemuse, vessie, gros ventre», budèt, budètch, budèytch «intestin, boyau» (Mistral, 1979 [1878]; Palay, 1980 [1932]), boda «gros ventre», bodega «cornemuse», bodena «gros ventre» (Alibert, 1977), pourraient être comparées avec les désignations du ver de terre mentionnées plus haut. Du point de vue phonétique, l'évolution BOT-> bod- est régulière avec changement de l'occlusive de sourde à sonore en position intervocalique une fois que la base se soude à un suffixe. Une suffixation autre que - ELLUS - -ic - n'est pas inconnue dans l'aire gasconne. À son tour, la désignation sarde mère de la terre, qui du point de vue stratigraphique pourrait appartenir à la couche très archaïque des désignations parentélaires (Alinei, 1994), peut être rapprochée de l'image du nombril ou plutôt du cordon ombilical par lequel la mère nourrit l'enfant. De même, le ver de terre, fertilisant naturel, nourrit la terre telle une génitrice (Carpitelli, 2012).

Dans les départements de Gironde, des Landes et du Lot-etGaronne, nous relevons dans le THESOC les aires lexicales de [bu’ðik] / [bu'zik] / [bu'rik] «ver de terre». [bwi:t] / [bwi:c], enregistrées sur la côte sud de la Charente-Maritime, semblent coïncider avec ces dénominations. Nous proposons de rapprocher cet ensemble $\mathrm{du}$ sud-ouest des désignations gallo-romanes de Haute-Bretagne :

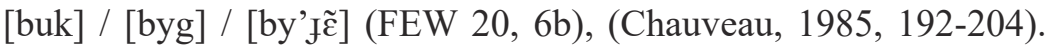
Ces dernières correspondent à leur tour aux zoonymes celtiques du Morbihan du type [byhy'Jet] qui sont eux-mêmes les variantes dialectales du groupe ['by:zyk] au nord de la Basse-Bretagne et du groupe ['by:ryk] du Sud-Finistère. Notons enfin la forme cornique bulligan attestée par Borlase à la fin du XVIII ${ }^{\mathrm{e}}$ siècle (Borlase, 1769).

La comparaison des formes celtiques et romanes du ver de terre met en évidence une concordance qui suggère une ancienne continuité atlantique. Cette continuité est d'abord rompue par la Manche, puis par le territoire s'étendant depuis la Loire jusqu'à l'île d'Oléron. Ce constat rappelle certains résultats déjà obtenus par Falc'hun lors de ses recherches. Il déclarait notamment : 
«Quand on compare le domaine bretonnant à l'ensemble des parlers romans de France d'après l'ALF, ce qui saute aux yeux, c'est le nombre de mots qui sont communs à tout ou partie du domaine occitan et au domaine bretonnant. Cette situation implique que ces mots communs ont jadis occupé une large bande côtière allant de la Basse-Bretagne aux Pyrénées; ils ont dû être refoulés par d'autres mots, qui ont descendu la Loire jusqu'à son embouchure, pour se propager ensuite entre Loire et Garonne.» (Falc'hun, 1985, 59)

À leur tour, les travaux de Le Dû et Brun-Trigaud ont plusieurs fois mis en évidence cette ancienne continuité atlantique (Le Dû et Brun-Trigaud, 2005 \& 2013) ${ }^{9}$.

Parmi les matériaux relatifs aux noms de la limace, on relève dans le sud-ouest de la France les désignations du type [mi' Kawko] et [Ki'mawk]. Ces derniers semblent à l'évidence apparentés. Un procédé métathique relierait les deux types même s'il paraît difficile de déterminer quelle serait la séquence originelle. [ $\mathrm{Ki}$ 'mawk] est répertorié dans le FEW sous l'entrée LIMAX. Cependant l'évolution de LIMAX sous la forme [Ki'mawk] est phonétiquement et morphologiquement problématique.

En s'appuyant sur les études de Jud (1941-42) et de Hubschmid (Vox Romanica 1938, 3, 86 cité dans FEW 5, 262b), on peut décomposer [mi' Kawko] à partir des éléments MIL- + *lauk(k)a. MIL- est d'origine celtique et signifie "animal, créature, petite bête» en irlandais, breton, gallois et cornique. ${ }^{*} \operatorname{lauk(k)a~renvoie~à~l'idée~de~}$ lumière, de brillance et de blancheur. Le motif de [mi' Kawko] pourrait donc être «animal brillant» en référence à la trace brillante laissée par la bave de l'animal. [mi' Kawko] concorde morpho-sémantiquement avec le gallois [malwodən] (Thomas 1973) et le breton [mel'hwe:dən] (Le Roux, 1924-1963). *lauk(k)a éluciderait également les formes romanes d'oil [li'k $\mathrm{j}][/[\mathrm{l} \jmath \mathrm{J}]$ «limace» rencontrées dans l'ouest de la France.

En breton, les ichtyonymes leog, laog, ['le:ok]/ ['la:ok] «arénicole» (Le Berre, 2008) supposeraient eux aussi un radical *leuk-/*lauk- «lumière, brillance». Rappelons en effet que l'aréni-

9. Voir aussi D. LE BRIS (dir.), Aires linguistiques /aires culturelles - Études de concordances en Europe occidentale : zones Manche et Atlantique, 2012. 
cole peut laisser sur la peau une sécrétion fluorescente quand on le manipule. Les formes celtiques armoricaines sont elles-mêmes apparentées aux appellations de ce ver marin en irlandais lugach (Mac an Iomaire, 2000), en gaélique d'Écosse lugas (Wentworth, 2003), en anglais dialectal de Cornouailles, lug, loog [lu:g] (Morton Nance, 1963). Précisons enfin que l'équivalent anglais $l u g$ est généralement présenté comme un emprunt au celtique.

Les désignations celtiques du type [gwrax] «vieille, labre», labrus berggylta, sont regroupées de manière assez homogène dans les extrémités occidentales de Grande-Bretagne (gallois gwrach et anglais dialectal cornique wrah) et d'Armorique (breton gwrah). Le motif de [gwrax] est transparent et signifie « vieille femme, sorcière, magicienne». Les celtophones de la zone Manche-Atlantique semblent avoir attribué à ce poisson des aptitudes surnaturelles. Dans certains contes, il peut apparaître comme une créature dotée d'un pouvoir magique qui lui permet de régner sur tous les autres animaux marins de la zone rocheuse où il se trouve (Sébillot, 1882, 272).

Le mot anglais wrasse est un emprunt au celtique. Les formes romanes vra, vras, vrac, vraichot, du type vra- (FEW 18, 133b, FEW 20, 17b ; Lepelley, 2005), recueillies en Normandie et en Haute-Bretagne depuis la côte du Bessin jusqu'à la Baie de Saint-Brieuc, sont elles aussi d'origine celtique. Elles remontent probablement à une période où les parlers gaulois étaient employés par les populations de ces territoires, c'est-à-dire au moins à l'Âge des métaux. Les formes anglaises et romanes sont aujourd'hui opaques pour leurs locuteurs. La prégnance motivationnelle et la concordance morpho-sémantique de [gwrax] constatées dans les péninsules galloise, cornique et armoricaine laissent supposer qu'il s'agirait ici de la forme lexicale la plus ancienne répertoriée dans cette zone pour nommer cette espèce de labridé.

L'analyse des dénominations bretonnes morgaz, morhad, morgad de la seiche, sepia officinalis, semblent identifiables au composé $m o r+k a z=\left\langle\right.$ mer + chat $»$ ou $m o r+$ gad $=\left\langle\right.$ mer + lièvre ${ }^{10}$.

10. En breton, dans ce genre de composé, le complément du nom mor- « mer » est antéposé. L'initiale consonantique du mot postposé est généralement lénifiée. Ainsi, pour reprendre les exemple cités, mor + kaz devient morgaz ; mor + gad, morhad. 
À l'est de la zone celtique de la Péninsule armoricaine, les termes margate, morgate, relevés depuis l'embouchure de la Seine jusqu'à Saint-Quay-Portrieux et depuis Le Croisic jusqu'à l'estuaire de la Gironde, montrent une nouvelle fois une continuité morpho-sémantique frappante entre les aires celtique et romane. Il semblerait là encore qu'il ne s'agit pas d'un phénomène d'emprunt explicable par des contacts linguistiques récents, mais davantage de rémanences d'une zone celto-atlantique remontant probablement à la Préhistoire. L'étude des dénominations celtiques et romanes morhast/morache «chien de mer, requin peau-bleue» dans cette même zone maritime (Le Bris, 2013) conduit à une conclusion très similaire.

La comparaison des aires celtiques et romanes et parfois germaniques, établie à partir du modèle des analyses motivationnelles réalisées dans le cadre de l'ALE et de l'ALiR, propose de véritables perspectives de recherches très prometteuses. En effet, le croisement et/ou la complémentarité dans certains cas des domaines roman et celtique permet parfois d'éclaircir des formes lexicales devenues opaques dans la première aire linguistique, mais toujours transparentes dans la seconde et vice-versa. Il met aussi à jour l'existence d'anciennes continuités linguistiques et culturelles qui, jusqu'à présent, ont été peu ou pas assez étudiées.

\section{Bibliographie}

AIS - JABERG Karl et JUD Jakob, Sprach- und Sachatlas Italiens und der Südschweiz, Zofingen, Ringier, 8 volumes, 1928-1940.

ALBB - LE RouX Pierre, Atlas Linguistique de Basse-Bretagne, ParisRennes, Plihon-Hommay, 1924-1963.

ALE - Atlas Linguarum Europae (1983-1990), vol. 1-4, Van Gorcum, Assen-Maastricht, 1997-2008, vol. 5-7, Rome, Istituto Poligrafico e Zecca dello Stato.

ALF - GiLliéron Jules et EDMONT Edmond, Atlas Linguistique de la France, Paris, Champion, 1902-1910. 
ALFMB - LE BERRE Alain, Atlas Linguistique de la Faune Marine de Bretagne, Brest, CRBC, 2008.

ALiR - Atlas Linguistique Roman, Rome, Istituto Poligrafico e Zecca dello Stato, 1986-...

ALIBERT Louis, Dictionnaire occitan-français d'après les parlers languedociens, Toulouse, IEO, 1966.

AlineI Mario, «Arc-en-ciel», Atlas Linguarum Europae, I, 1, cartes 6-9 et commentaire : 47-80, Assen, 1983.

AlineI Mario, «L'Atlas Linguarum Europae: risultati, struttura, storia, prospettive», in Pilar García Mouton (ed.), Geolingüistica. Trabajos europeos, Madrid, CSIC, 1994, p. 1-39.

Alinei Mario, L'origine delle parole, Roma, Aracne, 2009.

ALINEI Mario, «Dal latino pre-romano, attraverso i dialetti "moderni”, al latino di Roma : l'origine del lat. LUMBRICUS 'lombrico' dal lat. UMBILICUS », Quaderni di Semantica 2, 2010a, p. 177-188.

ALINEI Mario, «From pre-Roman Latin through "modern" dialects: the origins of Lat. lumbricus 'earthworm' from Lat. umbilicus 'navel'», in Gianni Belluscio et Antonio Mendicino (éds), Scritti in onore di Eric Pratt Hamp per il suo 90. Compleanno, Rende, Università della Calabria, 2010b, p. 3-13.

Alinei Mario et BArros FerreirA Manuela, "Coccinelle», Atlas Linguarum Europae, I, 4, cartes 42-44 et commentaire : 99-199, Assen-Maastricht, 1994.

Alvar Manuel, Léxico de los marineros peninsulares, Madrid, Arco/ Libros, 1985-1989.

BENOZzO Francesco, «La flora, la fauna, il paesaggio: l'importanza dei nomi dialettali per la conoscenza del passato preistorico», in Benozzo F. (éd.), Dizionario del Dialetto di San Cesario sul Panaro, vol. 2, San Cesario sul Panaro, Amministrazione Comunale, 2007, p. 7-39.

BORLASE William, Antiquities, historical and monumental of the country of Cornwall [...], Londres, Bowyer \& Nichols, 1769.

BRUN-Trigaud Guylaine, LE BERRE Yves et LE DÛ Jean, Lectures de l'Atlas Linguistique de la France de Gilliéron et Edmont, Paris, CTHS, 2005.

BRUN-TrIGAUD, Guylaine et LE DÛ Jean, «Les aires dialectales galloromanes à la lumière du celtique», Current Approaches to Limits and Areas in Dialectology, Carrilho E., Magro C. et Álvarez X. (éds.), New Castle Upon Tyne, Cambridge Scholars Publishing, 2013, p. 27-48. 
CARPITELli Elisabetta, «Retour sur le "ver de terre"... . Compléments occitans à la synthèse romane de l'Atlas Linguistique Roman", in Michèle Oliviéri, Guylaine Brun-Trigaud, Philippe Del Giudice, La leçon des dialectes. Hommages à Jean-Philippe Dalbera, Alessandria, Edizioni dell'Orso, 2012, p. 107-122.

CARPITELli Elisabetta et LE BRIS Daniel, «Concordances aréales an zone Atlantique», Current Approaches to Limits and Areas in Dialectology, Carrilho E., Magro C. et Álvarez X. (éds.), New Castle Upon Tyne, Cambridge Scholars Publishing, 49-68 + 5 cartes en annexe, 2013.

Chauveau Jean-Paul, «Quelques emprunts du gallo au breton», $L a$ Bretagne Linguistique 1, Brest, UBO-CRBC, 1985, p. 191-204.

Dalbera Jean-Philippe, Des dialectes au langage, Paris, Champion, 2006.

FALC'HUN François, «Le breton, forme moderne du gaulois», Annales de Bretagne 69, 1962, p. 413-428.

FALC'HUN François, Perspectives nouvelles sur l'histoire de la langue bretonne, Paris, UGE, 1981.

FALC'HUN François, «La structure linguistique du domaine bretonnant», La Bretagne Linguistique 1, 1985, p. 59-71.

FEW - WARTBURG Walter von, Französisches Etymologisches Wörterbuch, Basel, Zbinden Druck und Verlag AG, 1922 et suiv.

GADET Françoise, «Sociolinguistique, écologie des langues, et cetera», Langage et société, n. 129, 2009, p. 121-135.

Geiriadur Prifysgol Cymru, A Dictionary Of the Welsh Language, Cardiff, University of Wales, 2009.

JUD Jakob, Compte rendu de "Walter Hörz, Die Schnecke und Volkstum der Romanen. Diss., Tübingen, 1938", Vox Romanica 6, 19411942, p. 255-258.

KRUIJSEN Joep, «Quelques implications sémantiques d'une enquête linguistique européenne», in A. Varvaro (éd.), Atti del XIV Congresso Internazionale di Linguistica e Filologia Romanza, Napoli/ Amsterdam, Gaetano Macchiaroli / John Benjamins, 1977 [1974], p. 383-396.

LANAIA Alfio, «Il serpente-arcobaleno. Tradizioni e credenze popolari nella cultura classica», Quaderni di Semantica, 28, 2007, p. 187202.

LE BRIS Daniel, «Désignations bretonnes et romanes de la "seiche", sepia officinalis : concordances géo-linguistiques», in Michèle Oliviéri, Guylaine Brun-Trigaud, Philippe Del Giudice, La leçon 
des dialectes. Hommages à Jean-Philippe Dalbera, Alessandria, Edizioni dell'Orso, 2012, p. 159-172.

LE BRIS Daniel, «Concordances linguistiques celto-ibériques : les noms de "requin peau-bleue", prionace glauca, en Péninsule armoricaine et de "requin-taupe", lamna nasus, en Péninsule ibérique». Estudis Romanics [Institut d'Estudis Catalans] 35, 2013, p. 283-305.

LePelley René, Espèces marines de Normandie, Condé-sur-Noireau, Charles Corlet, 2005.

MAC An IOMAIRE Séamas, The Shores of Connemara (traduit de l'irlandais par Padraic de Bhaldraithe), Kinvara, Tír Eolas, 2000.

Mistral Frédéric, Dictionnaire provençal-français. Lou trésor dóu Félibrige, La Calade-Aix-en-Provence, Edisud, 2 vol., 1979 [1878]. Morton NANCE Robert, A Glossary Of Cornish Sea-Words, Marazion, Federation of Old Cornwall Societes, 1963.

Ríos PANISSE Maria del Carmen, Nomenclatura de la flora y fauna marítimas de Galicia, Anexos de Verba 7, 19, Saint-Jacques-deCompostelle, Universidade de Santiago de Compostela, 19771983.

Palay Simin, Dictionnaire du Béarnais et du Gascon modernes, Paris, CNRS, 1980 [1932].

SÉBILlot Paul, Les Traditions et superstitions de la Haute-Bretagne (réédité sous le titre Traditions, Croyances, Superstitions en HauteBretagne. L'Homme, les Esprits, les Démons, éditions club 35, 1998), Paris, Maisonneuve et Cie éditeurs, 1882.

ThOMAs Alan R., The linguistic geography of Wales: a contribution to Welsh dialectology, Cardiff, Univeristy of Wales Press, 1973.

Vitorino Gabriela, Atlas Linguístico do Litoral Português, Lisbonne, CLUL, 1985-...

Wentworth Roy, Gaelic Words and Phrases from Wester Ross, Inverness, Clàr, 2003. 
